\title{
Engaging Adolescents: Animal Assisted Therapy for Adolescents with Psychiatric and Substance Use Disorders
}

\author{
Kate C. Trujillo $\mathbb{1}^{1,2} \cdot$ Greg T. Kuo $^{1,2} \cdot$ Madelyne L. Hull ${ }^{1,2} \cdot$ Amanda E. Ingram ${ }^{1,2} \cdot$ Christian C. Thurstone $^{1,2}$
}

Published online: 2 October 2019

(c) The Author(s) 2019

\begin{abstract}
Objectives This study compares treatment engagement and outcomes between two groups of adolescents with psychiatric and substance use disorders (SUD): one received treatment with Animal Assisted Therapy (AAT) and another without AAT. Methods This is an exploratory study with a quasi-experimental pre-post comparison group design. Participants were 31 adolescents (ages 12-17 years) participating in an urban school-based substance treatment program. Cases $(n=14)$ received treatment with AAT, whereas comparison $(n=17)$ received treatment without AAT. The outcome measures included: number of sessions attended, proportion of negative urine drug screens for non-nicotine substances, overall-wellbeing, and the School Engagement Instrument. Student's T test and a Wilcoxon Rank Sum Test were used to evaluate differences. Outcomes that differed significantly between groups were further evaluated using unadjusted linear regression.

Results Participants who received treatment with AAT $(\mathrm{M}=9.9, \mathrm{SD}=3.2)$ attended more therapy sessions within a 16week period $(\beta=3.5, \mathrm{t}(1)=2.89, p=0.0072)$ than those that received treatment without AAT $(\mathrm{M}=6.4, \mathrm{SD}=3.5)$. Additionally, the AAT group (Mdn $=2.9$, IQR: $1.8,5.0)$ had a greater improvement in overall-wellbeing $(\beta=3.6, \mathrm{t}(1)=$ $3.74, p=0.0009)$ than treatment group without AAT (Mdn $=0.5$, IQR: $-2.6,1.4)$.

Conclusions AAT could be a way to increase engagement for youth in substance use treatment.
\end{abstract}

Keywords Animal assisted therapy (AAT) - Adolescent substance use disorder treatment $\cdot$ Mental health treatment $\cdot$ Therapeutic engagement $\cdot$ Co-occurring disorders

Incorporating animal-assisted therapy into adolescent mental health and substance treatment in a school-based setting may improve treatment access, engagement, retention, and outcomes. There is a major gap in treatment services for adolescents with substance use problems, especially those with co-occurring psychiatric disorders. The National Survey on Drug Use and Health estimates about 1 million adolescents aged 12-17 needed substance use treatment in 2017 , or $4.1 \%$ of adolescents in this age range (Center for Behavioral Health Statistics and Quality 2016). At the same time, only $8.8 \%$ of these youth received inpatient or outpatient substance use treatment (Center for Behavioral Health Statistics and Quality 2016). Studies also estimate

Kate C. Trujillo

ktruji21@msudenver.edu

1 Metropolitan State University of Denver, PO Box 173362, Denver, CO, USA

2 Denver Health, Denver, CO, USA that among adolescents with SUDs, 35-90\% have cooccurring mental health disorders, known as dual diagnoses (Hawkins 2008; Winstanley et al. 2012). Adolescents with dual diagnoses have higher rates of suicide, polysubstance use, delinquent and criminal activity, and out-of-home placements compared to adolescents with SUDs alone (Hawkins 2008).

To improve treatment utilization rates among adolescents with substance use problems, clinical models need to overcome current barriers to accessing treatment. These barriers include practical concerns such as lack of comfort, convenience, readiness, time and transportation (Kataoka et al. 2010; Mensinger et al. 2006). One way to remove barriers to treatment access for adolescents with substanceuse disorders may be to bring mental health and substance treatment into schools (Kataoka et al. 2010; New Freedom Commission on Mental Health 2003). By removing some of these barriers, school-based treatment may improve treatment access and completion rates, leading to better clinical outcomes. School-based mental health care has been proposed as a way to reduce health disparities for minority 
youth in particular (Bear et al. 2014, Committee on School Health 2004; Kataoka et al. 2010).

Unfortunately, even for adolescents who have overcome access barriers and are engaged in SUD treatment, current treatment models are unsatisfactory. For treatment of SUDs among adolescents, models that combine Motivational Enhancement Therapy (MET), Cognitive Behavioral Therapy (CBT), and Contingency Management (CM) have the most consistent empirical support (Dennis et al. 2004; Stanger et al. 2015). For treating adolescents with dual diagnoses, research supports the efficacy of four types of integrated treatment models: case management, MET, CBT, and family therapies (Brown et al. 2015; Hawkins 2008). However, even these models currently demonstrate low engagement, retention, and poor outcomes. For example, a study of 600 teenagers in outpatient substance treatment found that even the most empirically supported treatment models have dropout rates between $21-40 \%$ (Dennis et al. 2004). As with adult substance treatment (Gates et al. 2016), studies of adolescent substance treatment have found low and unstable abstinence rates and small effect at 9month follow-up (Hawkins 2008; Stanger et al. 2015). Compared to adolescents with SUDs alone, those with dual diagnoses have even poorer treatment outcomes due to poor attendance, poor compliance with treatment, and high rates of early termination (Hawkins 2008).

One way to improve treatment outcomes among adolescents may be to increase treatment engagement. Therapeutic engagement is defined by Welsh and McGrain (2008) as "a client's active involvement in and commitment to treatment" (p. 272). For adolescents in substance use disorder treatment, engagement predicted retention, and retention was correlated with better treatment outcomes (Abdel-Salam and Gunter 2014; Burrow-Sánchez et al. 2015). One strategy to improve treatment engagement and outcomes for adolescent mental health and SUD treatment may be to incorporate animal assisted therapy (AAT) (KernGodal et al. 2015; Stefanini et al. 2015).

Animal assisted therapy is defined as a goal-directed intervention by a health or human service professional with specialized expertise, practicing within his or her scope of practice, and partnering with an animal that meets specific criteria (Fine 2010). Psychotherapists who have used animals in therapy report that the animals promoted rapport in the therapeutic relationship (Corson and Corson 1980) and had a calming effect on clients (Odendaal and Meintjes 2003). Psychotherapists select animals for a therapeutic intervention based on the client, the therapeutic goal, and the attributes that the animal brings to the interaction (Trujillo et al. 2010).

However, few empirical studies explore whether incorporating AAT into adolescent mental health and SUD treatment may help improve treatment engagement, and consequently, treatment retention and outcomes. Studies of AAT in child and adolescent mental health treatment show mixed-yet promising-outcomes. One study with an empirical design that used AAT with children in outpatient mental health treatment. Shuck et al. (2015) $(n=24)$ found that group therapy with canine-assisted interventions resulted in a greater reduction in ADHD symptoms. There are four studies with an experimental design using AAT in adolescent inpatient mental health treatment (Balluerka et al. 2015; Muela et al. 2017; Seivert et al. 2018; Stefanini et al. 2015). Three of these studies found that incorporating AAT into treatment was associated with improvements in a variety of treatment outcomes: global functioning, social skills, school attendance, school adjustment, reduction in depression, reduction in hyperactivity and attention problems, and reduction in length of inpatient hospital stay (Balluerka et al. 2015; Muela et al. 2017; Stefanini et al. 2015). However, two of these studies also found that the AAT group was no better in terms of clinical symptoms than the control group (Balluerka et al. 2015; Stefanini et al. 2015). One of these studies (Seivert et al. 2018), found no significant differences in outcomes between the AAT and non-AAT group.

There are far fewer studies of AAT in adolescent substance treatment, and no studies with an experimental design that evaluate AAT in outpatient adolescent substance treatment. There is one study of AAT and adolescents in residential substance treatment. This study $(n=108)$ found that incorporating horse-assisted therapy (HAT) into treatment was associated with better treatment completion rates (Kern-Godal et al. 2015).

Using AAT to improve treatment engagement may be especially important for Hispanic youth because they have poorer treatment engagement and are more likely to terminate treatment early than non-Hispanic Whites (Huey 1998, McCabe 2002; Reyno and McGrath 2006 as cited in Kapke and Gerdes, 2016). We found three studies that used AAT with Hispanic youth. Only one of these studies compared outcomes between Hispanic and non-Hispanic youth (Shultz et al. 2007). This pilot study $(n=63)$ used a convenience sample where $51 \%$ of participants were nonHispanic White, $46 \%$ were Hispanic White, and 3\% were Black (Shultz et al. 2007). The Global Assessment of Functioning (GAF) scores of all participants improved after treatment, and there was no statistically significant difference between Hispanic and non-Hispanic participants for GAF score improvement (Shultz et al. 2007). The other two studies also showed successful outcomes when using AAT with Hispanic youth (Seivert et al. 2018; Shuck et al. 2015). Other non-randomized studies have found no significant difference between Hispanic and non-Hispanic adolescents (Black, 2012) or Hispanic and non-Hispanic adult pet owners regarding emotional attachment to their pet (Schoenfeld-Tacher et al. 2010). 
As we continue to develop our program to meet the needs of adolescents, primarily Hispanic, in a large urban community, we want to understand how including AAT with our standard treatment could affect our outcomes. This is an exploratory study with a post-hoc, quasi-experimental, pre-post comparison group design that evaluates an innovation, AAT, as an adjunct to treatment. This study compares two groups of adolescents with dual diagnoses in school-based outpatient mental health and substance treatment. This study compares clients who received treatment as usual (TAU) and TAU in addition to AAT by treatment participation, clinical indicators pre- and post-intervention, and sobriety rates. The primary hypothesis of this study is that AAT will be associated with increased treatment participation. The secondary hypothesis is that AAT will also be associated with improved clinical outcomes like overall well-being, school engagement, and sobriety. This study was approved by the Institutional Review Board. This study used retrospective, de-identified, secondary data to examine both groups.

\section{Method}

\section{Participants}

This study compares two groups of adolescents from an integrated healthcare clinic located inside a low-income urban, public secondary school. This school's student population ranges from 14 to 19 years of age, and most are from Spanish-speaking homes. The student population is 92\% Hispanic, 7\% African-American, and 0.7\% White; $79.3 \%$ are English Language Learners, and $93.3 \%$ of students are eligible for free or reduced lunch.

This study used a convenience sample of 31 urban middle and high school students referred to the therapist for substance use treatment and mental health counseling. The following demographic and clinical characteristics of the groups were recorded: age, gender, ethnicity, race, substance use disorder diagnoses, and psychiatric disorder diagnoses (Table 1). Students were assigned to groups (AAT or no AAT) by their academic class schedule and availability during the school day. (The therapy dog was available for two days each week, so if a client were available on a day that the dog was available, the AAT option would be the default choice, unless there was a reason to exclude this client from AAT.) The inclusion criteria for our study were: (1) ages 14 to 19 years; (2) ability to understand and provide written, informed parental consent and minor assent to receive substance treatment if under 15 years of age, or individual consent if 15 years of age or older; (3) DSM-V (Diagnostic and Statistical Manual of Mental Disorders) diagnosis of at least one non-nicotine
Table 1 Patient demographic and clinical characteristics, by animalassisted therapy (AAT) exposure group $(n=31)$

\begin{tabular}{|c|c|c|}
\hline & $\begin{array}{l}\text { No animal assisted } \\
\text { therapy }(n=17)\end{array}$ & $\begin{array}{l}\text { Animal assisted } \\
\text { therapy }(n=14)\end{array}$ \\
\hline $\mathrm{Age}^{\mathrm{a}}$ & $15.2+1.4$ & $13.7+1.4$ \\
\hline \multicolumn{3}{|l|}{ Gender $^{\mathrm{b}}$} \\
\hline Male & $9(53)$ & $11(79)$ \\
\hline Female & $8(47)$ & $3(21)$ \\
\hline \multicolumn{3}{|l|}{ Ethnicity $^{\mathrm{b}}$} \\
\hline Hispanic & $16(94)$ & $13(93)$ \\
\hline Non-Hispanic & $1(6)$ & $1(7)$ \\
\hline \multicolumn{3}{|l|}{ Race $^{b}$} \\
\hline White & 0 & $1(7)$ \\
\hline Black & $1(6)$ & 0 \\
\hline Other & $16(94)$ & $13(93)$ \\
\hline \multicolumn{3}{|c|}{ Substance use disorder diagnoses ${ }^{\mathrm{b}}$} \\
\hline Cannabis use disorder & $14(82)$ & $13(93)$ \\
\hline Alcohol use disorder & $10(59)$ & $5(36)$ \\
\hline Hallucinogen use disorder & $1(6)$ & 0 \\
\hline Stimulant use disorder & 0 & 0 \\
\hline Opioid use disorder & 0 & 0 \\
\hline $\begin{array}{l}\text { Other substance use } \\
\text { disorder }\end{array}$ & $1(6)$ & $1(7)$ \\
\hline \multicolumn{3}{|l|}{ Psychiatric disorders ${ }^{\mathrm{b}}$} \\
\hline Major depressive disorder & $9(53)$ & $9(64)$ \\
\hline $\begin{array}{l}\text { Generalized anxiety } \\
\text { disorder }\end{array}$ & $13(77)$ & $5(36)$ \\
\hline $\begin{array}{l}\text { Posttraumatic stress } \\
\text { disorder }\end{array}$ & $9(53)$ & $7(50)$ \\
\hline $\begin{array}{l}\text { Attention-deficit/ } \\
\text { hyperactivity disorder }\end{array}$ & $2(12)$ & $5(36)$ \\
\hline Conduct disorder & 0 & $1(7)$ \\
\hline $\begin{array}{l}\text { Oppositional defiant } \\
\text { disorder }\end{array}$ & $1(6)$ & 0 \\
\hline Social anxiety disorder & 0 & 0 \\
\hline Other & 0 & 0 \\
\hline
\end{tabular}

${ }^{\mathrm{a}}$ Mean + standard deviation

${ }^{\mathrm{b}} n(\%)$

SUD; and (4) willingness to participate in motivational interviewing (MI) and acceptance and commitment therapy (ACT). Exclusion criteria were: (1) mental illness that cannot be managed in an outpatient level of care; and (2) SUD that cannot be managed in an outpatient level of care. Exclusion criteria for the AAT group were: (1) fear of dogs; (2) allergies to dogs; (3) religious concerns about working with animals. (Note: Although exclusion criteria existed, none of the clients who were assigned to the AAT group were excluded from receiving AAT intervention.)

The demographic and clinical characteristics of the adolescent sample groups are described in Table 1. Overall, both groups were predominately Hispanic and presented for 
cannabis use disorder. All youth presented with a cooccurring psychiatric disorder. Most demographic and clinical characteristics of the groups appear similar, except gender, Alcohol Use Disorder, and Generalized Anxiety Disorder (Table 1). The experimental group was predominantly male (79\%), while the comparison group was evenly divided by gender. The comparison group had higher rates of Alcohol Use Disorder (59\%) and Generalized Anxiety Disorder (77\%) than the experimental group (36\%).

\section{Procedure}

Participants in this study received mental health and substance treatment at their school. Both client groups worked with the same therapist, a licensed clinical social worker and certified addictions counselor III. The therapist received 8 hours of formal Acceptance and Commitment Therapy (ACT) training and ongoing expert group supervision with a trainer certified by the Association of Contextual Behavioral Science. Both groups received manual-standardized motivational interviewing and acceptance and commitment therapy, called impACT (Thurstone 2018; Thurstone et al. 2017a). This model consists of 12 weeks of individual motivational interviewing, acceptance and commitment therapy, contingency management with urine drug screens, medication assisted therapy, family sessions and case management (Thurstone 2018; Thurstone et al. 2017a).

In addition to the standard treatment, the comparison group ( $n=14)$ received AAT with a certified therapy dog, an eight-year-old Labrador retriever. The dog and therapist have been certified by an independent agency as a therapy team. The certification process includes behavioral testing of the dog for desirable qualities of a dog that will be part of a professional setting and the therapist-handler's ability to work with the dog. These behaviors include walking on a loose leash, remaining calm in the presence of a loud or disruptive person, and remaining focused on the human handler in the presence of other dogs, among others (PTDC 2019). The certification process also uses the American Kennel Club's "Good Citizen" test for basic obedience and manners (AKC 2019). The dog was also under the continuous care of a veterinarian, fully vaccinated, and approved for work with adolescents in a mental health setting. Finally, a dog trainer also made quarterly visits to the therapy site to assess the dog and therapist team to ensure that the dog continued to be suitable to work as a therapy dog.

The therapist used manual-standardized ACT-informed AAT interventions in each session. These AAT interventions incorporate the therapy dog in mindfulness and communication exercises that help the client practice the six core ACT processes (Ingram 2017; Ladegard et al. 2017).
For example, in one session where clients are learning how to cope with substance cravings, the client will ask the dog to lie down on one side of the room and tell the dog to "stay." Then, the client will lay numerous unhealthy treats all over the floor between the dog and him/herself. The client walks to the opposite side of the room and then calls the dog to come to him/her. The dog must come to the client and ignore the unhealthy treats. The client then rewards the dog with healthy treats. Then the client and the therapist process how the dog managed the cravings and how this is similar or different to the client's experience. This exercise incorporates all six ACT core processes: (1) the client's self-awareness of his/her thoughts, feelings and behaviors around cravings; (2) the client's committed actions and specific skills used in managing cravings and making healthy choices; (3) the client's acceptance of the craving instead of fighting it; (4) the client defusing from temptation, and, instead, focusing on his/her values and healthier options; (5) connecting adolescents to their values and helping them to stay focused on their values even when they have cravings; (6) the client being mindful and in the present moment while making decisions.

\section{Measures}

The dependent variables of this study were: treatment participation, clinical outcomes, and sobriety. Data were collected for the first possible 16-week treatment period beginning from each individual's start date, up to the $12^{\text {th }}$ session. Engagement was measured by number of sessions attended. Clinical outcomes were measured by changes in client overall well-being and school engagement scores. For feasibility, client overall well-being was measured by one item from the Outcome Rating Scale (Owen et al. 2016): "Overall, I am doing well," on a scale from 0 (poor) to 10 (very good). School engagement was measured by the School Engagement Instrument (SEI), which consists of three items that assess behavioral, cognitive, and emotional dimensions of school engagement (Lintz et al. 2019). The three items were: "I followed the rules at school;" "I cared about what I'm learning in school;" and "I liked school;" which were scored on scales from 0 (definitely no) to 10 (definitely yes) for an overall score that ranged from 0 to 30 . Cronbach's alpha for SEI was 0.8 which suggests that the scale has acceptable internal consistency. During the client's intake, and at the beginning of each therapy session, the client completed the overall well-being and SEI scales. For the overall well-being and SEI, the intake measures were used as pre-treatment values. If these were unavailable, we used first therapy session values. The posttreatment values consisted of measures taken from the last session the client participated in during the 16-week time frame. Sobriety was measured by the proportion of negative 
urine drug screens (UDS). During the client's intake and at the end of every session, the client provides a UDS. Clients used the restroom in the school-based health clinic and were monitored by a nurse. The UDS were tested for amphetamines, benzodiazepines, cocaine, methamphetamine, opiates, and tetrahydrocannabinol (THC).

We also administered a brief survey to the clients in the AAT group to gain insight into their experience working with the therapy dog. After their 12th therapy session, clients were given a survey consisting of three questions rated on a Likert scale $(1=$ strongly disagree; $5=$ strongly agree): (1) Having the dog in my session makes me more willing to go to therapy; (2) Having the dog in my session makes me more comfortable being here; (3) Having the dog in my session makes me feel more willing to talk and trust the therapist; and two open-ended questions: (1) How has working with the therapy dog been helpful for you? (2) What could change to make working with the therapy dog better?

The therapist input the assessment data from these instruments and client baseline diagnoses, age, gender, and ethnicity into a database. For this study, all data were deidentified. Random identifiers for each client were generated by the clinical team.

\section{Data Analyses}

Data were analyzed with SAS Enterprise Guide 5.1 (SAS Institute, Inc. 2013). Depending on tests of normality, continuous data are presented as means with standard deviation or as medians with inter-quartile range; categorical variables are presented as number counts with percent. We evaluated differences in cases and controls using a
Student's T test and, for non-parametric data, a Wilcoxon Rank Sum Test. All tests were two-tailed. To reduce the chance of obtaining false positive results due to multiple comparisons in the study sample, we used a Bonferroniadjusted $p$-value of 0.0125 to detect statistical significance. If differences were detected and a linear relationship was observed, we then used simple linear regression to evaluate effect size.

\section{Results}

We compared intervention outcome measures based on exposure to AAT and observed statistically significant differences in the number of sessions attended $(p=0.0072)$ and the change in overall well-being $(p=0.0014)$ (Table 2). Improvement in school engagement scores for participants that received AAT was more than double the comparison group's, and although it was not statistically significant, it appears to be a trend. The difference in sobriety between groups was not statistically significant.

Unadjusted linear regression models revealed that compared to patients who received treatment without AAT $(\mathrm{M}=6.4, \mathrm{SD}=3.5)$, the AAT group $(\mathrm{M}=9.9, \mathrm{SD}=3.2)$ had a greater number of sessions completed within a 16week period $(\beta=3.5, \mathrm{t}(1)=2.89, p=0.0072)$. Additionally, the AAT group ( $\mathrm{Mdn}=2.9, \mathrm{IQR}: 1.8,5.0)$ had a larger improvement in overall well-being score $(\beta=3.6, \mathrm{t}(1)=$ 3.74, $p=0.0009)$, compared to treatment without AAT $(\mathrm{Mdn}=0.5$, IQR: $-2.6,1.4)($ Table 2$)$.

Qualitative feedback also indicated that the dog contributed to a positive experience and encouraged engagement in therapy (Table 3). Eight of the 14 clients in the AAT
Table 2 Differences in intervention outcome measures based on exposure to animalassisted therapy (AAT)

\begin{tabular}{lllll}
\hline & $\begin{array}{l}\text { No animal assisted } \\
\text { therapy }(n=17)\end{array}$ & $\begin{array}{l}\text { Animal assisted } \\
\text { therapy }(n=14)\end{array}$ & Test statistic & $p$-value \\
\hline Sessions completed $^{\mathrm{a}}$ & $6.4+3.5$ & $9.9+3.2$ & $\mathrm{t}(29)=-2.9$ & 0.0072 \\
Change in overall wellbeing $^{\mathrm{b}}$ & $0.5(-2.6,1.4)$ & $2.9(1.8,5.0)$ & $\mathrm{S}=133.0$ & 0.0014 \\
Change in school engagement $^{\mathrm{a}}$ & $2.4(0,1.0)$ & $6.6(4.9,10.0)$ & $\mathrm{S}=164.0$ & 0.0746 \\
$\begin{array}{l}\text { Proportion of total negative urine } \\
\text { drug screens }\end{array}$ & $0.7+0.4$ & $0.6+0.4$ & $\mathrm{t}(29)=0.4$ & 0.6956 \\
\hline
\end{tabular}

${ }^{\mathrm{a}}$ Mean + standard deviation

${ }^{\mathrm{b}}$ Median (IQR)
Table 3 Mean responses to the survey about the dog's presence for the animal assisted therapy (AAT) group

\begin{tabular}{ll}
\hline Question & Mean $(N=14)$ \\
\hline Having the dog in my session makes me more willing to go to therapy & \\
Having the dog in my session makes me more comfortable being here & $4.93+0$ \\
Having the dog in my session makes me feel more willing to talk and trust the therapist $^{\mathrm{a}}$ & $4.93+0.26$ \\
\hline
\end{tabular}

Likert scale ( $1=$ strongly disagree; $5=$ strongly agree)

${ }^{\mathrm{a}}$ Mean + standard deviation 
group reported the therapy dog helped them feel more "comfortable" or "safe" in therapy. One client said, "She's sweet; when she sees you cry she will be right there to lick your face and make you feel better. She is patient with you." Ten clients responded with "nothing" when asked what could change to make working with the therapy dog better. One client responded, "to cuddle more," and two clients commented that she should stop begging or sniffing.

\section{Discussion}

This study has immediate relevance and implications for treating Hispanic adolescents with co-occurring substance use and mental health disorders. Incorporating AAT into treatment was associated with better treatment outcomes, client engagement, and clients reported a high level of appreciation for the presence of the therapy dog. Strengths of this study include: (1) the sample came from a primarily low-income Hispanic population which is underrepresented in AAT research literature (Risley-Curtiss et al. 2006); (2) this study used a mix of self-reported and objective measures; and (3) because the same clinician worked with both groups, there was likely little inter-rater bias.

Our findings suggest there should be further research on using AAT to increase engagement for youth in substance and mental health treatment. Our study supports the feasibility of studying the incorporation of AAT into the treatment of adolescents with dual diagnoses, particularly Hispanic youth. Our sample had co-occurring psychiatric and substance use disorders $(100 \%)$ and was primarily Hispanic (94\%). We find this especially encouraging as Hispanic children have poorer treatment engagement and are more likely to terminate treatment early than nonHispanic Whites (Huey 1998, McCabe 2002, Reyno and McGrath 2006 as cited in Kapke and Gerdes 2016).

Increasing treatment accessibility, utilization, and engagement for youth with co-occurring disorders is of utmost importance. Yet engagement in treatment is complicated and can be broadly grouped into two categories, behavioral (showing up) and attitudinal (being "ready" for change) (Staudt 2007). Our findings lay the basis to examine engagement with AAT in conjunction with SUD and mental health intervention, particularly in the context of schoolbased settings. We measured engagement behaviorally, by session attendance. However, the critical question remains: does this engagement in the treatment process ultimately lead to long-term sobriety or improved mental health?

\section{Limitations and Future Research}

There are various limitations to this study. First, because this study lacked random assignment, but rather, used a comparison group, the data were confounded and we are unable to conclude that differences among groups can be attributed to AAT as part of treatment. There were also demographic and clinical differences between groups. Specifically, the non-AAT group was evenly split between boys and girls, while the AAT group was predominately male. Also, the non-AAT group had higher rates of alcohol use disorder, generalized anxiety disorder, and a lower rate of attention-deficit/hyperactivity disorder compared to the AAT group. The scope of the study was also limited by the following factors: small sample size, limited external validity due to single site intervention, and treatment by one therapist. For these reasons, the results of this study may not generalize to different settings. The study was also designed post-hoc. No evaluation was done to assess therapist's adherence to either treatment model. The sample's socioeconomic backgrounds and psychiatric medications were not recorded. Most of the outcome measures were selfreported and completed in the presence of the therapist, so they may have been affected by social desirability bias. Additional measures such as teacher assessments of school engagement were not used due to limitations in funding. While the Outcome Rating Scale is a reliable and valid instrument (Bringhurst et al. 2006), only a single scale from the instrument was used in this study; partial use of the Outcome Rating Scale has not been studied.

Future research should address this study's limitations by randomizing the participants in each group and having a larger, more diverse sample from multiple sites with multiple therapists. Recordings of sessions should also be made and assessed to ensure adherence to MI, ACT, and AAT. Engagement should also be measured both behaviorally and attitudinally to more richly describe the experience of the intervention and the mechanism by which youth relate their interactions with the therapy dog/therapist team, and changes in their substance use and mental health. School engagement should be measured by objective measures like student attendance and teacher assessment. Also, qualitative questions related to understanding how AAT with Hispanic youth supports the clinical process would add depth to the findings.

Acknowledgements The authors wish to thank the Denver Health Foundation and the Department of Social Work at Metropolitan State University of Denver for their support of this project. The authors would also like to acknowledge the contributions of Michaela Hennig to this article.

\section{Compliance with Ethical Standards}

Conflict of Interest The authors declare that they have no conflict of interest. The authors alone are responsible for the content and writing of the paper. The authors do wish to avoid any perceived conflict. One author, who owns and directs a for-profit agency devoted to training 
and certifying clinicians working with dogs in mental health, developed the AAT interventions used in our study.

Ethical Approval All procedures performed in studies involving human participants were in accordance with the ethical standards of two institutional research committees (Colorado Multiple Institutional Review Board, IRB registration number: IORG0000433; Metropolitan State University of Denver Institutional Review Board, IRB registration number: IRB00005388) and with the 1964 Helsinki declaration and its later amendments or comparable ethical standards. All applicable international, national, and/or institutional guidelines for the care and use of animals were followed.

Publisher's note Springer Nature remains neutral with regard to jurisdictional claims in published maps and institutional affiliations.

Open Access This article is distributed under the terms of the Creative Commons Attribution 4.0 International License (http://crea tivecommons.org/licenses/by/4.0/), which permits unrestricted use, distribution, and reproduction in any medium, provided you give appropriate credit to the original author(s) and the source, provide a link to the Creative Commons license, and indicate if changes were made.

\section{References}

Abdel-Salam, S., \& Gunter, W. D. (2014). Therapeutic engagement as a predictor of retention in adolescent therapeutic community treatment. Journal of Child \& Adolescent Substance Abuse, 23 (1), 49-57.

AKC. (2019). American Kennel Club- Canine Good Citizen Test. AKC https://www.akc.org/products-services/training-programs/ canine-good-citizen/.

Balluerka, N., Muela, A., Amiano, N., \& Caldentey, M. A. (2015). Promoting psychosocial adaptation of youths in residential care through animal-assisted psychotherapy. Child Abuse \& Neglect, 50, 193-205. https://doi.org/10.1016/j.chiabu.2015.09.004.

Bear, L., Finer, R., Guo, S., \& Lau, A. S. (2014). Building the gateway to success: an appraisal of progress in reaching underserved families and reducing racial disparities in school-based mental health. Psychological Services, 11(4), 388.

Black, K. (2012). The relationship between companion animals and loneliness among rural adolescents. Journal of Pediatric Nursing, 27(2), 103-112.

Bringhurst, D. L., Watson, C. W., Miller, S. D., \& Duncan, B. L. (2006). The reliability and validity of the outcome rating scale: a replication study of a brief clinical measure. Journal of Brief Therapy, 5(1), 23-30.

Brown, R. A., Abrantes, A. M., Minami, H., Prince, M. A., Bloom, E. L., Apodaca, T. R., \& Matsko, S. V. (2015). Motivational interviewing to reduce substance use in adolescents with psychiatric comorbidity. Journal of Substance Abuse Treatment, 59, 20-29.

Burrow-Sánchez, J. J., Meyers, K., Corrales, C., \& Ortiz-Jensen, C. (2015). The influence of cultural variables on treatment retention and engagement in a sample of Mexican American adolescent males with substance use disorders. Psychology of Addictive Behaviors, 29(4), 969.

Center for Behavioral Health Statistics and Quality (2016). Key substance use and mental health indicators in the United States: Results from the 2015 National Survey on Drug Use and Health (HHS Publication No. SMA 16-4984, NSDUH Series H-51). Rockville, MD: Center for Behavioral Health Statistics and Quality, Substance Abuse and Mental Health Services Administration. http://www.samhsa.gov/data/.
Committee on School Health (2004). School-based mental health services. Pediatrics, 113(6), 1839-1845.

Corson, S. A., \& Corson, E. O. (1980). Pet animals as nonverbal communication mediators in psychotherapy in institutional settings. In S. A. Corson \& E. O. Corson (Editors) Ethology and nonverbal communication in mental health: an interdisciplinary biopsychosocial exploration. New York, NY: Pergamon Press.

Dennis, M., Godley, S. H., Diamond, G., Tims, F. M., Babor, T., Donaldson, J., \& Funk, R. (2004). The Cannabis Youth Treatment (CYT) study: main findings from two randomized trials. Journal of Substance Abuse Treatment, 27(3), 197-213. https:// doi.org/10.1016/j.jsat.2003.09.005.

Fine, A. H. (2010). Handbook on animal-assisted therapy: theoretical foundations and guidelines for practice. Amsterdam; Boston: Elsevier/Academic.

Gates, P. J., Sabioni, P., Copeland, J., Le Foll, B., \& Gowing, L. (2016). Psychosocial interventions for cannabis use disorder. In Cochrane database of systematic reviews. John Wiley \& Sons, Ltd. https://doi.org/10.1002/14651858.CD005336.pub4.

Hawkins, E. H. (2008). A tale of two systems: co-occurring mental health and substance abuse disorders treatment for adolescents. Annual Review of Psychology, 60(1), 197-227. https://doi.org/10. 1146/annurev.psych.60.110707.163456.

Ingram, A 2017). impACT animal assisted therapy manual (Unpublished working manual). Denver, CO: Pawsitive Connections.

Kapke, T. L., \& Gerdes, A. C. (2016). Latino family participation in youth mental health services: treatment retention, engagement, and response. Clinical Child and Family Psychology Review, 19(4), 329-351.

Kataoka, S., Novins, D. K., \& Santiago, C. D. (2010). The practice of evidence-based treatments in ethnic minority youth. Child and Adolescent Psychiatric Clinics, 19(4), 775-789.

Kern-Godal, A., Arnevik, E. A., Walderhaug, E., \& Ravndal, E. (2015). Substance use disorder treatment retention and completion: a prospective study of horse-assisted therapy (HAT) for young adults. Addiction Science \& Clinical Practice, 10, 21 https://doi.org/10.1186/s13722-015-0043-4.

Ladegard, K., Ingram, A., \& Benningfield, M. (2017). An innovative model for integrating substance use treatment in schools. The Brown University Child and Adolescent Behavior Letter, 33(1), 1-7. https://doi.org/10.1002/cbl.30182.

Lintz, M., Thurstone, C., Hull, M., \& Ladegard, K. (2019). Development of a schoolbased substance treatment model. Journal of Child and Adolescent Substance Abuse. (Accepted for publication).

Mensinger, J. L., Diamond, G. S., Kaminer, Y., \& Wintersteen, M. B. (2006). Adolescent and therapist perception of barriers to outpatient substance abuse treatment. The American Journal on Addictions, 15(1), s16-s25.

Muela, A., Balluerka, N., Amiano, N., Caldentey, M. A., \& Aliri, J. (2017). Animal-assisted psychotherapy for young people with behavioural problems in residential care. Clinical Psychology \& Psychotherapy, 24(6), O1485-O1494.

New Freedom Commission on Mental Health (2003). Achieving the Promise: Transforming Mental Health Care in America. Final Report. Rockville, MD: New Freedom Commission on Mental Health. http://govinfo.library.unt.edu/mentalhealthcommission/ reports/FinalReport/downloads/InsideCover.pdf.

Odendaal, J. S., \& Meintjes, R. A. (2003). Neurophysiological correlates of affiliative behaviour between humans and dogs. The Veterinary Journal, 165(3), 296-301.

Owen, J., Miller, S. D., Seidel, J., \& Chow, D. (2016). The working alliance in treatment of military adolescents. Journal of Consulting and Clinical Psychology, 84(3), 200-210.

PTDC (2019). Professional therapy dogs of colorado test. (Unpublished Exam). Denver, CO: PTDC.

Risley-Curtiss, C., Holley, L. C., \& Wolf, S. (2006). The animalhuman bond and ethnic diversity. Social Work, 51(3), 257-268. 
SAS Institute Inc. (2013). Base SAS 9.4 Utilities: Reference. Cary, NC: SAS2.

Schoenfeld-Tacher, R., Kogan, L. R., \& Wright, M. L. (2010). Comparison of strength of the human-animal bond between Hispanic and non-Hispanic owners of pet dogs and cats. Journal of the American Veterinary Medical Association, 236(5), 529-534.

Schuck, S. E., Emmerson, N. A., Fine, A. H., \& Lakes, K. D. (2015). Canine-assisted therapy for children with ADHD: preliminary findings from the positive assertive cooperative kids study. Journal of attention disorders, 19(2), 125-137.

Schultz, P. N., Remick-Barlow, G. A., \& Robbins, L. (2007). Equineassisted psychotherapy: a mental health promotion/intervention modality for children who have experienced intra-family violence. Health \& Social Care in the Community, 15(3), 265-271.

Seivert, C., Casey, J., \& May (2018). Animal assisted therapy for incarcerated youth: RCT. Applied Developmental Science, 22(2), 139-153.

Stanger, C., Ryan, S. R., Scherer, E. A., Norton, G. E., \& Budney, A. J. (2015). Clinic- and home-based contingency management plus parent training for adolescent cannabis use disorders. Journal of the American Academy of Child \& Adolescent Psychiatry, 54(6), 445-453.e2. https://doi.org/10.1016/j.jaac.2015.02.009.

Staudt, M. (2007). Treatment engagement with caregivers of at-risk children: Gaps in research and conceptualization. Journal of Child and Family Studies, 16(2), 183-196.
Stefanini, M. C., Martino, A., Allori, P., Galeotti, F., \& Tani, F. (2015). The use of animal-assisted therapy in adolescents with acute mental disorders: a randomized controlled study. Complementary Therapies in Clinical Practice, 21(1), 42-46. https:// doi.org/10.1016/j.ctcp.2015.01.001.

Thurstone, C. (2018). Introducing ImpACT for adolescents: a training manual for mental health professionals. Retrieved from http:// drthurstone.com/impact-introduction/.

Thurstone, C., Hull, M., Timmerman, J., \& Emrick, C. (2017a). Development of a motivational interviewing/acceptance and commitment therapy model for adolescent substance use treatment. Journal of Contextual Behavioral Science.

Trujillo, K., Tedeschi, P., \& Williams, J. (2010). Research meets practice: Issues for evidence-based training in HAI. In P. McCardle, S. McCune, L. Esposito, J. Griffin, \& L. Freund (Editors), The role of pets in children's lives: human-animal interaction in child development, health, and therapeutic intervention (pp. 119-215). Baltimore, MD: Brookes Publishing.

Welsh, W. N., \& McGrain, P. N. (2008). Predictors of therapeutic engagement in prison-based drug treatment. Drug \& Alcohol Dependence, 96(3), 271-280.

Winstanley, E. L., Steinwachs, D. M., Stitzer, M. L., \& Fishman, M. J. (2012). Adolescent substance abuse and mental health: problem co-occurrence and access to services. Journal of Child \& Adolescent Substance Abuse, 21(4), 310-322. https://doi.org/10. 1080/1067828X.2012.709453. 